\title{
Discovery of a low-mass companion inside the debris ring surrounding the F5V star HD 206893
}

\author{
J. Milli $^{1}$, P. Hibon ${ }^{1}$, V. Christiaens ${ }^{2,3,4}$, É. Choquet $^{5}$, M. Bonnefoy ${ }^{6}$, G. M. Kennedy ${ }^{7}$, M. C. Wyatt ${ }^{7}$, O. Absil ${ }^{4}$, \\ C. A. Gómez González ${ }^{4}$, C. del Burgo ${ }^{8}$, L. Matrà ${ }^{7}$, J.-C. Augereau ${ }^{6}$, A. Boccaletti ${ }^{9}$, C. Delacroix ${ }^{10}$, S. Ertel $^{11}$, \\ W. R. F. Dent ${ }^{12}$, P. Forsberg ${ }^{13}$, T. Fusco ${ }^{14}$, J. H. Girard ${ }^{1}$, S. Habraken ${ }^{4}$, E. Huby ${ }^{4}$, M. Karlsson ${ }^{13}$, A.-M. Lagrange ${ }^{6}$, \\ D. Mawet ${ }^{5,16}$, D. Mouillet ${ }^{6}$, M. Perrin ${ }^{15}$, C. Pinte ${ }^{6}$, L. Pueyo ${ }^{15}$, C. Reyes ${ }^{1}$, R. Soummer ${ }^{15}$, J. Surdej ${ }^{4}$, \\ Y. Tarricq ${ }^{1}$, and Z. Wahhaj ${ }^{1}$ \\ (Affiliations can be found after the references)
}

Received 15 October 2016 / Accepted 28 November 2016

\begin{abstract}
Aims. Uncovering the ingredients and the architecture of planetary systems is a very active field of research that has fuelled many new theories on giant planet formation, migration, composition, and interaction with the circumstellar environment. We aim at discovering and studying new such systems, to further expand our knowledge of how low-mass companions form and evolve.

Methods. We obtained high-contrast $H$-band images of the circumstellar environment of the F5V star HD 206893, known to host a debris disc never detected in scattered light. These observations are part of the SPHERE High Angular Resolution Debris Disc Survey (SHARDDS) using the InfraRed Dual-band Imager and Spectrograph (IRDIS) installed on VLT/SPHERE.

Results. We report the detection of a source with a contrast of $3.6 \times 10^{-5}$ in the $H$-band, orbiting at a projected separation of 270 milliarcsec or $10 \mathrm{au}$, corresponding to a mass in the range 24 to $73 M_{\text {Jup }}$ for an age of the system in the range 0.2 to 2 Gyr. The detection was confirmed ten months later with VLT/NaCo, ruling out a background object with no proper motion. A faint extended emission compatible with the disc scattered light signal is also observed.

Conclusions. The detection of a low-mass companion inside a massive debris disc makes this system an analog of other young planetary systems such as $\beta$ Pictoris, HR 8799 or HD 95086 and requires now further characterisation of both components to understand their interactions.
\end{abstract}

Key words. brown dwarfs - circumstellar matter - planet-disk interactions - planetary systems

\section{Introduction}

Through direct imaging, instruments fed with adaptive optics (AO) have enabled the detection and characterisation of a few tens of low-mass companions, either giant planets (hereafter GP) or brown dwarfs (BD), probing a parameter space in the mass vs orbital radius still inaccessible with other indirect techniques such as radial velocities or transits. The direct detection of the thermal emission of such substellar objects brings precious information for understanding their formation mechanisms and physical properties (see Bowler 2016, for a recent review). In addition, many of the GP/BD systems discovered in high-contrast imaging are associated to a debris disc, generally detected through its infrared or submillimetre emission (e.g. HR 8799, Marois et al. 2006; HD 95086, Rameau et al. 2013; HR 3549, Mawet et al. 2015; HR 2562, Konopacky et al. 2016). In only three cases, this disc was also resolved in scattered light ( $\beta$ Pictoris, HD 106906 and Fomalhaut), enabling to study the interactions with the companion.

This letter presents the discovery of a low-mass BD in orbit around the nearby F5V star HD 206893 located at $38.3 \pm$ $0.8 \mathrm{pc}$ (see details in Table 1). The star is known to host a debris disc detected through its large infrared excess $\left(L_{\text {dust }} / L_{\star}=\right.$ $2.3 \times 10^{-4}$, Moór et al. 2006), characterised through its Spectral Energy Distribution (SED) with Spitzer/IRS-MIPS (Chen et al. 2014), and marginally resolved with Herschel/PACS (as detailed in this letter). This study also presents the putative scattered light signal of the disc, at low signal-to-noise $(\mathrm{S} / \mathrm{N})$ due to the faintness of its emission. The age of the system is not well constrained as it is not known to belong to a moving group. Zuckerman \& Song (2004) estimated an age of 200 Myr based on $\mathrm{X}$ ray, radial velocity and proper motion measurements. This age is also inferred by Holmberg et al. (2009) with an upper limit of 1.2 Gyr from Padova stellar evolution models. More recently, David \& Hillenbrand (2015) suggest a median age of 2.1 Gyr using a Strömgren photometry fit to stellar atmosphere models in a Bayesian framework while Pace (2013) derives $860 \pm 710$ Myr based on the chromospheric activity calibrated against the Geneva-Copenhagen survey.

\section{Observation and data reduction}

The companion was first detected with VLT/SPHERE in 2015. HST/NICMOS archival data from 2007 showed that the companion cannot be a background object without proper motion and data from VLT/NaCo redetected the object in 2016.

\subsection{SPHERE}

The SPHERE High Angular Resolution Debris Disc Survey (SHARDDS) is a high resolution imaging survey aimed at resolving and characterising new debris discs never detected in scattered light (PI: Milli, 096.C-0388, 097.C-0394, see also Wahhaj et al. 2016). This programme is a search for discs around 
Table 1. Properties of the system HD 206893.

\begin{tabular}{lccc}
\hline \hline Property & HD 206893 & HD 206893 B & Ref. \\
\hline Distance (pc) & \multicolumn{2}{c}{$38.34 \pm 0.8$} & 1 \\
Proper motion & $\mu_{\alpha} \times \cos \delta=93.67 \pm 0.66$ & 1 \\
(mas/yr) & \multicolumn{2}{c}{$\mu_{\delta}=0.33 \pm 0.37$} & 1 \\
Age (Gyr) & \multicolumn{2}{c}{$0.2-2.1$} & 2 \\
Spectral type & F5V & L5-L9 dwarf & $1 / 3,4$ \\
$H$ mag & 5.69 & $16.79 \pm 0.06$ & $1 / 3$ \\
$L^{\prime}$ mag & 5.52 & $13.43_{-0.15}^{+0.17}$ & $1 / 4$ \\
Mass & $1.24 M_{\odot}$ & $24^{a} / 50^{b} / 73^{c} M_{\text {Jup }}$ & $5 / 3$ \\
$T_{\text {eff }}(\mathrm{K})$ & 6486 & $1200^{a} / 1310^{b} / 1380^{c}$ & $2 / 3$ \\
Separation (mas) & & $270.4 \pm 2.6$ & 3 \\
& & $268.8 \pm 10.4$ & 4 \\
PA ( $\left.{ }^{\circ}\right)$ & & $69.95 \pm 0.55$ & 3 \\
& & $61.6 \pm 1.9$ & 4 \\
\hline
\end{tabular}

Notes. For an age of ${ }^{(a)} 0.2 \mathrm{Gyr}^{\left({ }^{(b)}\right.} 0.8 \mathrm{Gyr}$, and ${ }^{(c)} 2$ Gyr respectively.

References. (1) van Leeuwen (2007); (2) Zuckerman \& Song (2004), Holmberg et al. (2009), Pace (2013), David \& Hillenbrand (2015); (3) this work: VLT/SPHERE $(05 / 10 / 15)$; (4) this work: VLT/NaCo (08/08/16); (5) David \& Hillenbrand (2015).

stars within $100 \mathrm{pc}$ having an infrared excess greater than $10^{-4}$, with the IRDIS subsystem (Dohlen et al. 2008) in broad band $\mathrm{H}$ and the apodised Lyot coronagraph of diameter 185 mas. Each target is observed in pupil-stabilised mode to allow angular differential imaging (ADI, Marois et al. 2006). On 5 October 2015, we observed the star HD 206893. Over the 40 min effective onsource integration time, we obtained $50^{\circ}$ field rotation. The atmospheric conditions were average with a mean seeing of $0.9^{\prime \prime}$ and a coherence time of $2.8 \mathrm{~ms}$, resulting in a Strehl ratio of $85 \%$ in the $H$-band. The raw frames were sky-subtracted, flat-fielded and bad-pixel corrected using the SPHERE Data Reduction and Handling pipeline (Pavlov et al. 2008), resulting in a temporal cube of 576 frames with individual integration time $4 \mathrm{~s}$. The frames were thereafter re-centred using the four satellite spots imprinted in the image during the centring sequence obtained before and after the 576 frames. With broad-band filters, these satellite spots are elongated. We fitted a 2D Gaussian to each spot and evaluated the star location as the intersection of the two lines joining the centres of opposite satellite spots, as explained in Wertz et al. (2017), which yields an absolute centring accuracy of $0.2 \mathrm{px}$ or 2.5 mas. The individual frames of the cube were not re-centred relative to one another because an active centring using the SPHERE differential tip-tilt sensor is dealing with this to an accuracy smaller than what can be obtained from an individual frame-to-frame recentring (Wertz et al. 2017). We reduced the images using the principal component analysis algorithm (PCA, Soummer et al. 2012), as implemented in the Vortex Image Processing pipeline (VIP ${ }^{1}$, Gomez Gonzalez et al. 2017, Fig. 1). In this algorithm, the only free parameter is the number of modes removed. We detect a point source with an $\mathrm{S} / \mathrm{N}$ of 14 (Fig. 1) at a projected separation of $270.4 \pm 2.6$ mas or 10.4 au.

\subsection{NICMOS}

HD 206893 was observed on 12 June 2007 with the NICMOS instrument on the Hubble Space Telescope (HST). We re-analysed the data (see Appendix A) and do not detect any point source.

\footnotetext{
Available at https://github.com/vortex-exoplanet/VIP
}

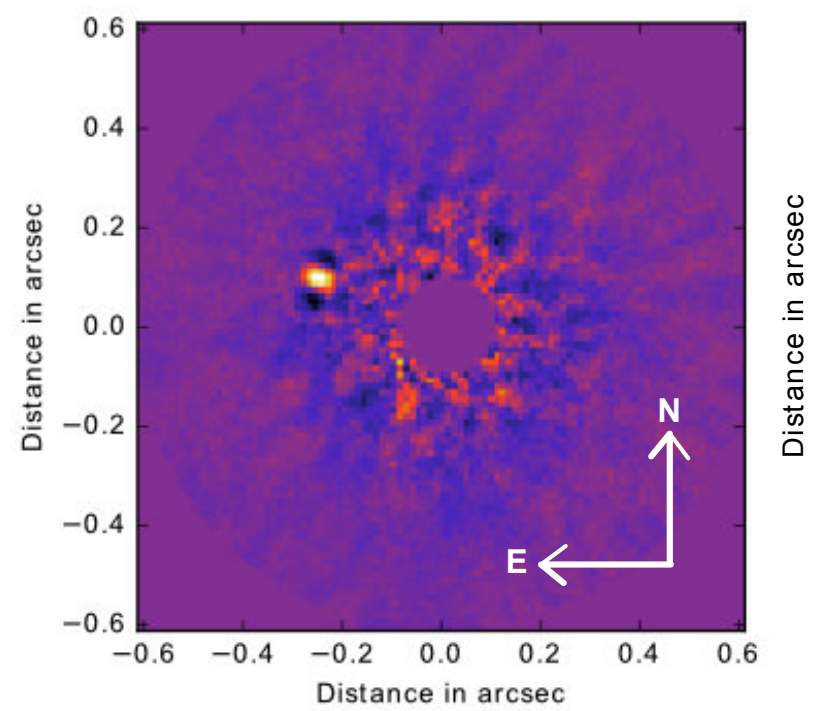

Fig. 1. SPHERE $H$-band coronagraphic image reduced with ADI + PCA, showing the detection of the companion HD 206893 B at 270 mas with a $\mathrm{S} / \mathrm{N}$ of 14 .

\section{3. $\mathrm{NaCo}$}

HD 206893 was observed with VLT/NaCo on 8 August 2016, taking advantage of its AGPM coronagraph (e.g. Mawet et al. 2005, 2013). This observation was part of programme 095.C0937(B) (PI: O. Absil). HD 206893 was observed in pupilstabilised mode using the $27.1 \mathrm{mas} /$ pixel plate scale and the $L^{\prime}$-band filter $(3.8 \mu \mathrm{m})$. The seeing $\left(0.7-0.8^{\prime \prime}\right)$ and the coherence time $(\sim 5 \mathrm{~ms})$ were stable throughout the observation. A total of 90 science data cubes of $0.3 \mathrm{~s}$ (DIT) exposure and 200 (NDIT) frames were obtained, corresponding to 1 h30 onsource and a $107^{\circ}$ total parallactic angle variation. Sky data cubes were obtained every 10-12 min. The star was carefully re-centred behind the coronagraph after each sky observation to a $\sim 0.2-0.3$ pixel accuracy. Four data cubes were also obtained with a shorter exposure time and the star offset from the coronagraph centre (but still behind the AGPM substrate), to obtain unsaturated PSF images. These data cubes were used for photometric calibration and to generate fake companions.

After standard calibrations (sky subtraction, flat-fielding and bad pixel correction), the frames were re-centred by fitting a negative Gaussian to the AGPM central hole as done in Absil et al. (2013). The frame selection process, essential to reach the best contrast, kept the 12879 most correlated frames and with the lowest level of residual speckle noise out of the 18000 original frames. These 12879 frames were binned four by four, to yield a final ADI cube of 3219 frames. The final cube was reduced using algorithms based on the PCA implemented in VIP, as shown in Fig. 2 with seven principal components removed, which was found to optimise the companion $\mathrm{S} / \mathrm{N}$ (approximately six).

\section{Analysis}

\subsection{Detection of the companion HD 206893 B}

The astrometry and photometry of the point source detected in the SPHERE and NaCo data set are described in Table 1. The ADI algorithms affect the astrometry and photometry of a detected companion. A robust way to estimate them is to use the negative fake companion algorithm (hereafter NEGFC; e.g. Lagrange et al. 2010). For both SPHERE and $\mathrm{NaCo}$, we used 

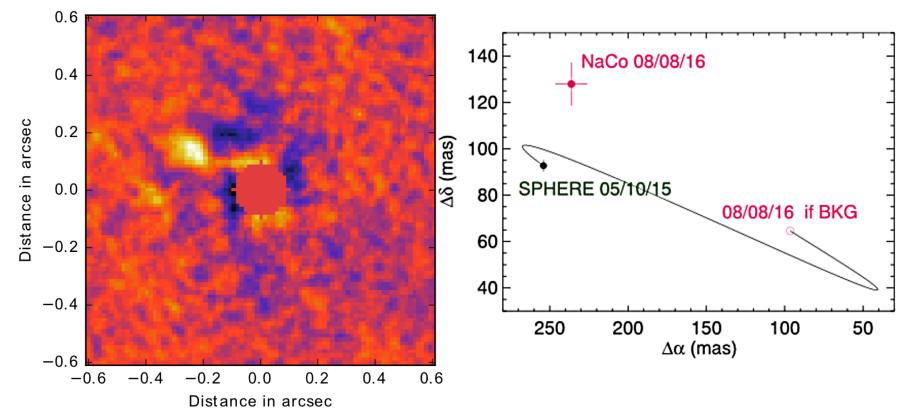

Fig. 2. Left: image of NaCo reduced with PCA-ADI after removing seven principal components, which was found to optimise the companion $\mathrm{S} / \mathrm{N}(\sim 6)$. Right: common proper motion analysis. The black line displays the motion of the companion if it was a background source. The uncertainty is given at $1 \sigma$.

NEGFC as implemented in VIP (Wertz et al. 2017) with the exploration of the three parameters (radial separation, PA and contrast) performed with a simplex algorithm minimising the residual standard deviation in an aperture at the location of the companion. The uncertainties given in Table 1 combine that on the instrument (plate-scale, north alignement, filter transmission), the centring uncertainty and the measurement uncertainty due to the presence of speckle noise, as detailed in Wertz et al. (2017) for SPHERE and Absil et al. (2013) for NaCo. We used a true north offset of $-1.75 \pm 0.08^{\circ}$ for SPHERE (Maire et al. 2016) and re-calibrated the $\mathrm{NaCo}$ true north against that of SPHERE using the common astrometric field 47 Tuc observed in September 2016 which yielded a value of $0.58 \pm 0.10^{\circ}$. The measurement uncertainty was computed by injecting fake companions at the separation of the point source and various azimuths, and by retrieving their astrometry and photometry with the NEGFC algorithm. It dominates the $\mathrm{NaCo}$ error budget. For SPHERE, the budget of error is dominated by the $2.5 \mathrm{mas}$ conservatively attributed to the centring accuracy. The contrast curves obtained from SPHERE, NaCo and NICMOS are shown in Fig. 3. They take into account the penalty term coming from small-sample statistics at small separations (Mawet et al. 2014).

This target is at high galactic latitude $\left(-44^{\circ}\right)$ and therefore a background contamination within $0.3^{\prime \prime}$ of the star is unlikely. To confirm the object is truly bound, we computed its expected position if it was a background object with no significant proper motion for the date corresponding to the NICMOS and $\mathrm{NaCo}$ data. We find a separation and PA of $\left(0.995^{\prime \prime} \pm 0.006^{\prime \prime} ; 85.3^{\circ} \pm\right.$ $\left.0.58^{\circ}\right)$ for the NICMOS epoch in 2007 and $\left(0.174^{\prime \prime} \pm 0.003^{\prime \prime}\right.$; $61.0^{\circ} \pm 0.6^{\circ}$ ) for the $\mathrm{NaCo}$ epoch in 2016 also shown in Fig. 2 right, using a star proper motion of $94.2 \mathrm{mas} / \mathrm{yr}$ at PA $89.9^{\circ}$. In the NICMOS data, no point source is detected at high confidence level at the position where the candidate would have been in 2007 assuming it is a background star. Figure 3 shows the $5 \sigma$ radial detection limit measured on the combined image. We also repeated the same processing steps described in Sect. 2.2 after injecting a synthetic NICMOS PSF in the raw data at the background star position and at the $3.6 \times 10^{-5}$ contrast measured on our candidate in the SPHERE data. The injected point source is detected at $7 \sigma$ as shown in Fig. A.1, demonstrating that the candidate found with SPHERE would have been detected at a high confidence level, if it were a background object. In addition, the star was also observed in two other exoplanet surveys: the International Deep Planet Survey (IDPS, Galicher et al. 2016) with the Gemini North/NIRI instrument and the Gemini Planet-finding campaign with the Subaru/NICI instrument

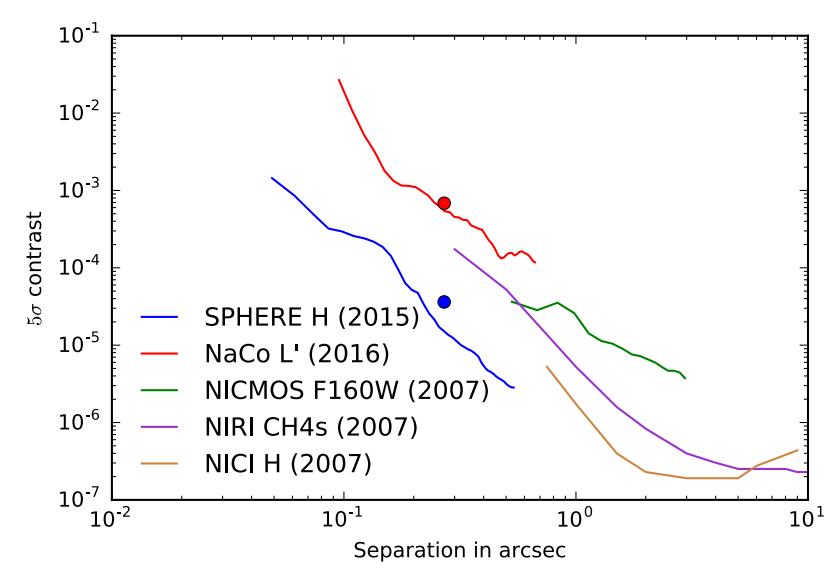

Fig. 3. Constrast curves at $5 \sigma$. The red and blue dots show the positions of the companion in the $\mathrm{NaCo}$ and SPHERE data respectively.

(Wahhaj et al. 2013). No detection is reported as their discovery spaces start from $0.3^{\prime \prime}$ and $0.5^{\prime \prime}$ respectively (Fig. 3) but they would have been sensitive to a background object with the same magnitude at $1^{\prime \prime}$.

In the $\mathrm{NaCo}$ data, the position of the companion is clearly not compatible within error bars with a background object (Fig. 2 right). We interpret the changes in projected separation and position angle between the IRDIS and $\mathrm{NaCo}$ data as due to the orbital motion of the companion (see Sect. 3.3). We can thus confidently assert that this object is bound to HD 206893.

\subsection{Companion physical properties}

The companion HD $206893 \mathrm{~B}$ has a very red colour, with $3.19_{-0.16}^{+0.18}$ mag difference between the $H$ and $L^{\prime}$-band. Figure 4 compares its position in a colour-magnitude diagram to that of other young companions and field dwarfs (Leggett et al. 2010, 2013). As the age is debated, we overplotted three isochrones using LYON evolutionary tracks (Chabrier et al. 2000; Baraffe et al. 2003) for $200 \mathrm{Myr}$, 800Myr and 2 Gyr. HD 206893 B lies among the L5-L9 field dwarf objects, with a similar $L^{\prime}$ magnitude as 2MASS 0122-2439 B (Bowler et al. 2013) but a redder colour. This makes it the reddest object among young and dusty L dwarfs in the field, which is likely due to a dusty atmosphere although interstellar or disc reddening cannot be ruled out. Using an age of $200 \mathrm{Myr}$ (respectively $800 \mathrm{Myr}$, 2 Gyr) and the AMES-Cond models (Baraffe et al. 2003), the $H$-band contrast of HD $206893 \mathrm{~B}$ implies an object of $24 M_{\text {Jup }}$ and effective temperature $1230 \mathrm{~K}\left(50 M_{\text {Jup }}\right.$ with $1330 \mathrm{~K}$, and $75 M_{\text {Jup }}$ with $1420 \mathrm{~K}$ respectively).

\subsection{Relation to the debris disc and orbital motion}

The debris disc is marginally resolved with Herschel/PACS with an inclination of $40^{\circ} \pm 10^{\circ}$ along the PA $60^{\circ} \pm 10^{\circ}$. This is presented in Appendix B along with a modelling concluding on a disc inner radius of $50 \mathrm{au}$. With a projected separation of $10.4 \pm 0.1 \mathrm{au}$, the companion appears therefore to be interior to the disc, with a PA consistent with an orbit in the same plane as the disc. As shown in Fig. 2 right, the companion moved during the 306 days separating the SPHERE and NaCo detections. We applied the methods laid in Pearce et al. (2015) to constrain the orbit of a companion imaged over short orbital arcs. With the two epochs, the linear sky motion is $0.05 \pm 0.01^{\prime \prime} / \mathrm{yr}$ at a position angle of $-27_{-17}^{\circ+13}$. We derived thereafter the parameter $B$ 


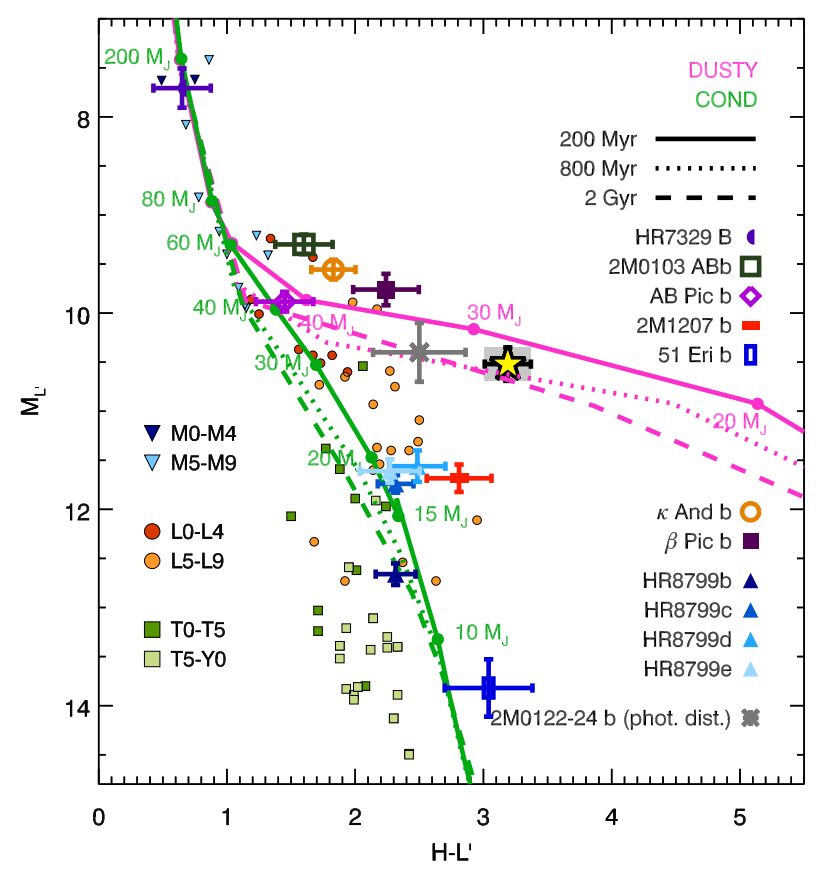

Fig. 4. Colour-magnitude diagram, obtained from Galicher et al. (2014) with the new photometry of HD $206893 \mathrm{~B}$ (yellow star), that of 51 Eri (Macintosh et al. 2015) and 2MASS 0122-2439 B (Bowler et al. 2013). The lines show the isochrones for different ages and evolution models.

and $\phi$ of Pearce et al. (2015), that proves useful to constrain the possible orbits. Including astrometric, distance, and $10 \%$ stellar mass uncertainties we find $B=0.33_{-0.14}^{+0.19}$ and $\phi=90^{\circ} \pm 15^{\circ}$. The constraints are weak as the $\mathrm{NaCo}$ astrometry is relatively poor; in particular we cannot constrain the ascending node to test whether the orbit is indeed coplanar with the disc. If we assume a low eccentricity orbit, the semi-major axis is about $12 \mathrm{au}$ (37 yr period). Conversely, for a high eccentricity, if the apocentre distance is restricted to be below 50 au (i.e. the approximate disc inner edge), this implies that the companion is currently at apocentre, that is with a semi-major axis below $10 \mathrm{au}$. If we restrict the companion to lie within a projected separation of 500 mas in June 2007 to avoid detection by NICMOS (see Fig. 3), the marginalised constraints on specific parameters do not improve much. If the companion truncates the disc, then the semi-major axis is expected to be $\sim 1 / 3$ of the inner edge radius (Holman \& Wiegert 1999), so $15-20$ au $\left(0.4-0.5^{\prime \prime}\right)$. Thus, while there is no evidence to suggest that the companion's orbit is unusual, more precise astrometry is required to constrain the orbital elements and draw conclusions about the relation between the companion's orbit and the disc.

This architecture is strikingly similar to the HR 2562 system (Konopacky et al. 2016), where a $30 \pm 15 M_{\text {Jup }}$ brown dward was detected at 20 au of its host star, within a debris disc of inner radius $\sim 75$ au.

\section{Conclusions}

This letter presents the detection of a low-mass companion orbiting at a projected separation of 10 au around the F5V star HD 206893 as part of the SHARDDS survey, thanks to VLT/SPHERE high contrast capabilities. The object was confirmed by VLT/NACO and proven not to be a background source by HST/NICMOS. With an $H$-band contrast of 11 mag, evolutionary models suggest the object could be a $25 M_{\text {Jup }}$ brown dwarf if the system is $200 \mathrm{Myr}$, or twice as massive for a $800 \mathrm{Myr}$ system. Along with its $L^{\prime}$ contrast of 7.9, the object appears very red, and the closest to L5-L9 field dwarfs in a colour-magnitude diagram. The orbital motion is detected and suggests an orbital period of $\sim 37 \mathrm{yr}$ in case of low eccentricty.

In addition, we report the detection of the disc, through its thermal emission with Herschel/PACS at a position angle of $\sim 60^{\circ}$ almost aligned with the projected position of the companion. This system is therefore reminiscent of the cases of HR 8799, HD 95086 or $\beta$ Pictoris where one of several GPs have been detected in orbit inside a Kuiper belt analog. It is the second brown dwarf detected in the inner hole of a debris disc after HR 2562.

Several aspects make this system very attractive for future characterisation. The contrast is well within range of current extreme AO instruments, enabling spectral identification. The orbital motion is fast enough to allow orbit monitoring which can bring constraints on the dynamical mass of the object. Deeper observations may detect the scattered light of the disc and confirm the faint emission seen in our image, to understand if the companion is responsible for the inner truncation of the disc at about $50 \mathrm{au}$, or possibly reveal asymetries and clumps resulting from interactions between the disc and the brown dwarf or possible yet undiscovered planets.

Acknowledgements. J.M. is supported by the ESO fellowship programme. He thanks R. Galicher for his re-reduction of the NIRI data, and O. Wertz for his help with VIP and NEGFC. E.C. is supported by NASA through Hubble Fellowship grant HST-HF2-51355 and HST-AR-12652 awarded by STScI, operated by the AURA, Inc., for NASA under contract NAS5-26555. O.A. is a F.R.S.-FNRS Research Associate. The research leading to these results was partly funded by the European Research Council under the European Union's Seventh Framework Programme (ERC Grant Agreement No. 337569), and by the French Community of Belgium through an ARC grant for Concerted Research Action. G.M.K. is supported by the Royal Society as a Royal Society University Research Fellow. M.C.W. and L.M. are supported by the European Union through ERC grant 279973. V.C. acknowledges J. Smoker and M. Espinoza for their help with $\mathrm{NaCo}$. V.C. is supported by the Millennium Science Initiative (Chilean Ministry of Economy) through grant RC130007. C.d.B. acknowledges support from the Mexican CONACyT research grant CB-2012-183007.

\section{References}

Absil, O., Milli, J., Mawet, D., et al. 2013, A\&A, 559, L12 Baraffe, I., Chabrier, G., Barman, T. S., Allard, F., \& Hauschildt, P. H. 2003, A\&A, 402, 701

Bowler, B. P. 2016, PASP, 128, 102001

Bowler, B. P., Liu, M. C., Shkolnik, E. L., \& Dupuy, T. J. 2013, ApJ, 774, 55 Chabrier, G., Baraffe, I., Allard, F., \& Hauschildt, P. 2000, ApJ, 542, 464

Chen, C. H., Mittal, T., Kuchner, M., et al. 2014, ApJS, 211, 25

Choquet, É., Perrin, M. D., Chen, C. H., et al. 2016, ApJ, 817, L2

David, T. J., \& Hillenbrand, L. A. 2015, ApJ, 804, 146

Dohlen, K., Langlois, M., Saisse, M., et al. 2008, in Ground-based and Airborne Instrumentation for Astronomy II, Proc. SPIE, 7014

Galicher, R., Rameau, J., Bonnefoy, M., et al. 2014, A\&A, 565, L4

Galicher, R., Marois, C., Macintosh, B., et al. 2016, A\&A, 594, A63 Gomez Gonzalez, C. A., Wertz, O., Absil, O., et al. 2017, ApJ, submitted Holman, M. J., \& Wiegert, P. A. 1999, AJ, 117, 621

Holmberg, J., Nordström, B., \& Andersen, J. 2009, A\&A, 501, 941

Kennedy, G. M., Wyatt, M. C., Sibthorpe, B., et al. 2012a, MNRAS, 421, 2264

Kennedy, G. M., Wyatt, M. C., Sibthorpe, B., et al. 2012b, MNRAS, 426, 2115 Konopacky, Q. M., Rameau, J., Duchêne, G., et al. 2016, ApJ, 829, L4 Lagrange, A.-M., Bonnefoy, M., Chauvin, G., et al. 2010, Science, 329, 57 Leggett, S. K., Burningham, B., Saumon, D., et al. 2010, ApJ, 710, 1627 Leggett, S. K., Morley, C. V., Marley, M. S., et al. 2013, ApJ, 763, 130 Macintosh, B., Graham, J. R., Barman, T., et al. 2015, Science, 350, 64 Maire, A.-L., Langlois, M., Dohlen, K., et al. 2016, ArXiv e-prints [arXiv: 1609.06681] 
Marois, C., Lafrenière, D., Doyon, R., Macintosh, B., \& Nadeau, D. 2006, ApJ, 641, 556

Mawet, D., Riaud, P., Absil, O., \& Surdej, J. 2005, ApJ, 633, 1191

Mawet, D., Absil, O., Delacroix, C., et al. 2013, A\&A, 552, L13

Mawet, D., Milli, J., Wahhaj, Z., et al. 2014, ApJ, 792, 97

Mawet, D., David, T., Bottom, M., et al. 2015, ApJ, 811, 103

Milli, J., Mouillet, D., Lagrange, A.-M., et al. 2012, A\&A, 545, A111

Moór, A., Ábrahám, P., Derekas, A., et al. 2006, ApJ, 644, 525

Moór, A., Pascucci, I., Kóspál, Á., et al. 2011, ApJS, 193, 4

Pace, G. 2013, A\&A, 551, L8

Pavlov, A., Feldt, M., \& Henning, T. 2008, in Astronomical Data Analysis Software and Systems XVII, ASP Conf. Series, 394, 581

Pearce, T. D., Wyatt, M. C., \& Kennedy, G. M. 2015, MNRAS, 448, 3679

Rameau, J., Chauvin, G., Lagrange, A.-M., et al. 2013, ApJ, 772, L15

Soummer, R., Pueyo, L., \& Larkin, J. 2012, ApJ, 755, L28

Soummer, R., Perrin, M. D., Pueyo, L., et al. 2014, ApJ, 786, L23

van Leeuwen, F. 2007, A\&A, 474, 653

Wahhaj, Z., Liu, M. C., Nielsen, E. L., et al. 2013, ApJ, 773, 179

Wahhaj, Z., Milli, J., Kennedy, G., et al. 2016, A\&A, 596, L4

Wertz, O., Absil, O., Gómez González, C. A., et al. 2017, A\&A, in press, DOI: $10.1051 / 0004-6361 / 201628730$

Zuckerman, B., \& Song, I. 2004, ARA\&A, 42, 685

1 European Southern Observatory (ESO), Alonso de Córdova 3107, Vitacura, Santiago, Chile

e-mail: jmilli@eso.org

2 Departamento de Astronomía, Universidad de Chile, Casilla 36-D, Santiago, Chile

3 Millenium Nucleus Protoplanetary Disks in ALMA Early Science, Santiago, Chile
${ }^{4}$ Space Sciences, Technologies and Astrophysics Research (STAR) Institute, Université de Liège, 19c allée du Six Août, 4000 Liège, Belgium

5 Jet Propulsion Laboratory, California Institute of Technology, 4800 Oak Grove Drive, Pasadena, CA 91109, USA

${ }^{6}$ Univ. Grenoble Alpes, CNRS, IPAG, 38000 Grenoble, France

7 Institute of Astronomy, University of Cambridge, Madingley Road, Cambridge, UK

8 Instituto Nacional de Astrofísica, Óptica y Electrónica, Luis Enrique Erro 1, Sta. Ma. Tonantzintla, Puebla, Mexico

${ }^{9}$ LESIA, Observatoire de Paris, PSL Research University, CNRS, Sorbonne Universités, UPMC Univ. Paris 06, Univ. Paris Diderot, Sorbonne Paris Cité, 5 place Jules Janssen, 92195 Meudon, France

${ }^{10}$ Sibley School of Mechanical and Aerospace Engineering, Cornell University, Ithaca, USA

11 Steward Observatory, University of Arizona, 933 N Cherry Ave, Tucson, AZ 85719, USA

12 Atacama Large Millimeter/submillimeter Array (ALMA) Santiago Central Offices, Alonso de Córdova 3107, Vitacura, Casilla 763 0355, Santiago, Chile

13 Department of Engineering Sciences, Ångström Laboratory, Uppsala University, PO Box 534, 75121 Uppsala, Sweden

14 ONERA, The French Aerospace Lab, BP72, 29 avenue de la Division Leclerc, 92322 Chatillon Cedex, France

15 Space Telescope Science Institute, 3700 San Martin Drive, Baltimore, MD 21218, USA

16 Department of Astronomy, California Institute of Technology, 1200 E. California Blvd, MC 249-17, Pasadena, CA 91125, USA 


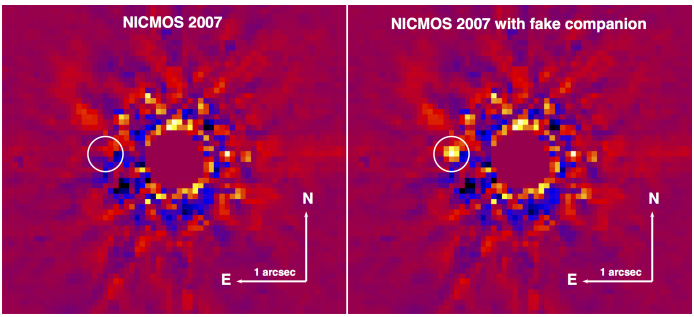

Fig. A.1. F160W NICMOS 2007 coronagraphic images, processed with RDI + PCA without injecting a fake companion (left $)$ and after injection of a fake companion of contrast $11.1 \mathrm{mag}$ at the location where the candidate would have been in 2007 assuming it is a background object with no proper motion (white circle).

\section{Appendix A: NICMOS non detection}

HD 206893 was observed on 12 June 2007 with the NICMOS instrument on HST, as part of a survey looking for debris discs around nearby stars (PI: J. Rhee, GO-11157). The data were obtained with the mid-resolution NIC2 channel (plate scale $0.07565^{\prime \prime} / \mathrm{px}$ ) with a coronagraph of radius $0.3^{\prime \prime}$, in two filters F160W and F110W. The target was observed at two orientations of the telescope separated by $\sim 30^{\circ}$, to enable PSF subtraction with roll differential imaging. We reprocessed the F160W archival dataset (centre wavelength $1.6006 \mu \mathrm{m}, \mathrm{FWHM}$ $0.4012 \mu \mathrm{m}$ ) with the same PSF subtraction method as used in the Archival Legacy Investigations for Circumstellar Environment (ALICE) programme (Soummer et al. 2014; Choquet et al. 2016). We used the KLIP algorithm (Soummer et al. 2012) on PSF libraries composed of images from multiple reference stars. After bad pixel correction, we selected the 454 images (from 78 reference stars) the most correlated with each of HD 206893's exposures, out of a reference star library assembled with the ALICE pipeline. This selection favoured images from 78 different stars chosen mostly from the two dominant HST programmes in the initial library (programmes 11157 and 10176). Figure A.1 (left) shows the combined image after subtracting synthetic PSFs computed from the 55 strongest eigenmodes of the library. No point source is detected at the position where the candidate would have been in 2007 if it were a background object without proper motion. Our detection limits (Figs. 3 and A.1 right show that the point source would have been detected in that case. This re-analysis shows that the point source is co-moving with the star.

\section{Appendix B: The disc around HD 206893}

Archival Herschel PACS data were obtained and modelled in the same way as Kennedy et al. (2012a,b). The $70 \mu \mathrm{m}$ image is shown in Fig. B.1 (top image). The subtraction of a scaled calibration observation (PSF) clearly shows that the source HD 206893 is not point-like (bottom image) and reveals the approximate disc extent and position angle. The debris disc is only a few beams across so constraints on the disc properties are relatively poor. Our disc best fitting model assumes a temperature $T_{\text {disk }}=288 r^{-0.4}$, with $r$ in au, and has a decreasing powerlaw surface density distribution of $\Sigma \propto r^{-0.5}$ extending from 50 to $200 \mathrm{au}$. This is compatible with previous modelling by Moór et al. (2011) who proposed a modified blackbody model for the disc with a temperature of $49 \mathrm{~K}$ and a radius of $49 \mathrm{au}$. This is also compatible with the colder $(48 \mathrm{~K})$ dust population in the double component disc model of Chen et al. (2014). The disc is inclined (from face-on) by about $40^{\circ}$ at a position angle of $\sim 60^{\circ}$ (East of North). The uncertainties on these angles are on the order of $10^{\circ}$.
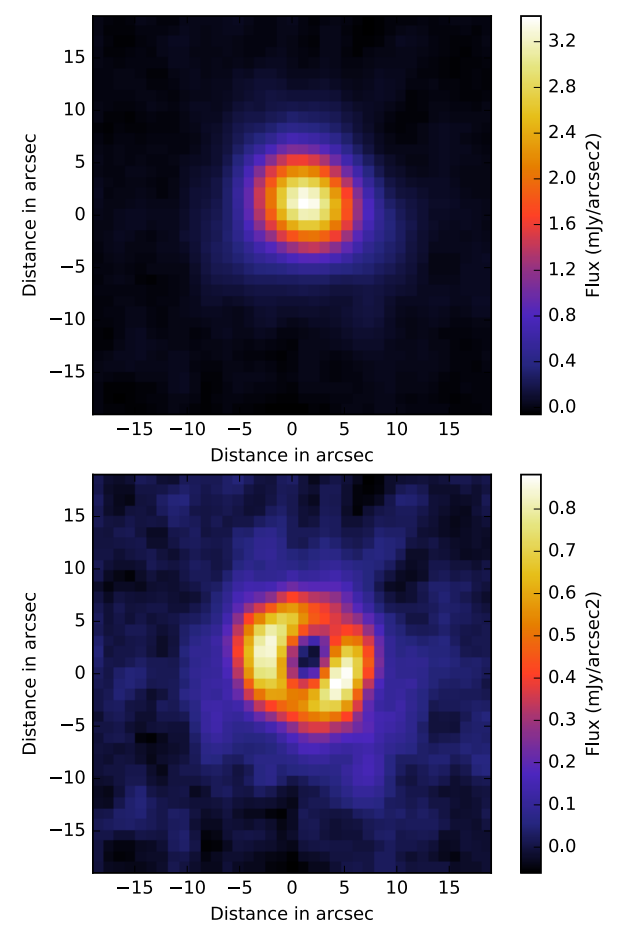

Fig. B.1. Herschel/PACS image of the star HD 206893 at $70 \mu \mathrm{m}$ (top). The bottom image shows the residuals after subtraction of the Herschel PSF, showing the source is not point-like.

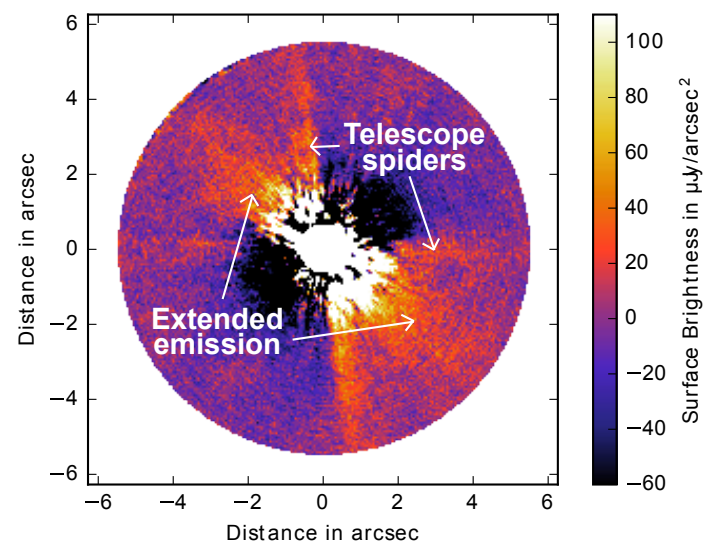

Fig. B.2. SPHERE $H$-band image in its complete $12^{\prime \prime} \times 12^{\prime \prime}$ field of view after a classical ADI reduction, in a linear colour scale in $\mu \mathrm{Jy} / \operatorname{arcsec}^{2}$. It shows a faint and extended emission along the same PA as the Herschel residual image likely coming from the disc.

To try to reveal the disc in scattered light, we reduced the SPHERE images using classical ADI (Fig. B.2 showing the whole $12^{\prime \prime} \times 12^{\prime \prime}$ field of view of SPHERE/IRDIS) to maximise the sensitivity in the backround and limit flux losses induced with more aggresive reductions (Milli et al. 2012) and binned the pixels by a factor two. We see a faint extended emission along the PA $\sim 60^{\circ}$ with a surface brighntess of $\sim 0.05 \mathrm{mJy} / \operatorname{arcsec}^{2}$. This faint emission is detected from $\sim 1.5^{\prime \prime}$ (60 au) up to $\sim 4-5^{\prime \prime}$ (150-190 au) where the background noise starts to dominate.We could confidently rule out spurious emission along that PA coming from the diffraction pattern from the spiders or the elongation of the PSF due to the wind at the ground level or at higher altitudes. Furthermore the PA is compatible with the Herschel/PACS residual image. We therefore tentatively attribute this signal to the scattered light of the debris disc with a $\mathrm{S} / \mathrm{N}$ of approximately one. 